\title{
BIOMETRIA DE FRUTOS E SEMENTES E GERMINAÇÃO DE Plathymenia reticulata BENTH. E Plathymenia foliolosa BENTH. (FABACEAE - MIMOSOIDEAE) ${ }^{1}$
}

\author{
Renata de Melo Ferreira Lopes², Valéria Lúcia de Oliveira Freitas² e José Pires de Lemos Filho³
}

\begin{abstract}
RESUMO - Este estudo objetivou comparar características biométricas de frutos e sementes e o efeito de diferentes escarificações, temperaturas e luz na germinação de Plathymenia reticulata Benth. e Plathymenia foliolosa Benth. Foram registrados comprimento, largura, espessura, massa da matéria seca e fresca de frutos $(n=100)$ e sementes ( $n=100$ ) de cada espécie. Os diferentes tratamentos foram escarificações mecânica e química e temperaturas (fotoperíodo/nictoperíodo) de 20,30 e $35 / 15^{\circ} \mathrm{C}\left(12 / 12\right.$ e 0/24) e 25 e $35^{\circ} \mathrm{C}(12 / 12)$. Os frutos de $P$. foliolosa mostraram-se mais largos, espessos e pesados e as sementes, mais compridas e espessas do que as de $P$. reticulata. As sementes de ambas as espécies não apresentaram fotoblastismo. A escarificação ácida não aumentou significativamente a germinabilidade das sementes em relação ao grupo-controle, enquanto a escarificação mecânica incrementou significativamente a germinabilidade apenas de $P$. foliolosa. As germinabilidades a $25^{\circ} \mathrm{C}$ das sementes de $P$. reticulata intactas, escarificadas com ácido e lixa foram, respectivamente, de 55\%, 60\% e 89\%. Para as sementes de P. foliolosa esses valores foram 48\%, 37,5\% e 83\%, respectivamente.Esses resultados apontam limitações na germinação de $P$. foliolosa impostas pelo tegumento, entretanto o efeito deste restringindo a germinação das sementes intactas decresceu com a elevação da temperatura.
\end{abstract}

Palavras-chave: Ecótipos, Temperatura e Escarificação.

\section{BIOMETRY OF FRUITS AND SEEDS AND GERMINATION OF Plathymenia reticulata BENTH. AND Plathymenia foliolosa BENTH. (FABACEAE - MIMOSOIDEAE)}

\begin{abstract}
This study aimed to compare the biometry of fruits and seeds, as well as the effect of different scarification, temperature and light conditions on the germination of Plathymenia reticulata Benth. and Plathymenia foliolosa Benth. We measured the length, width, thickness, and fresh mass of fruits $(n=100)$ and seeds $(n=100)$ of each specie. The different treatments were mechanic and acid scarification, temperatures (photoperiod/ nictoperiod) of 20,30 and $35 / 15^{\circ} \mathrm{C} \mathrm{(12/12} \mathrm{and} \mathrm{0/24)} \mathrm{and} 25$ and $35{ }^{\circ} \mathrm{C}(12 / 12)$. P. foliolosa fruits were wider, thickener and heavier. Its seeds are longer and thicker than those of $\boldsymbol{P}$. reticulata. Seeds of both species were not photosensitive. Acid scarification did not increase significantly the percentage of seed germination of both species, but mechanic scarification increased significantly the percentage of seed germination for $\boldsymbol{P}$. foliolosa. The percentage of germination at $25^{\circ} \mathrm{C}$ of intact seeds of $\boldsymbol{P}$. reticulata, scarified with acid and with sandpaper was, respectively, 55\%, 60\% and 89\%. For seeds of P. foliolosa values obtained were, respectively, $48 \%, 37.5 \%$ and $83 \%$. These results indicate limitations of germination of $\boldsymbol{P}$. foliolosa imposed by the tegument but the effect of tegument restringing the germination of intact seeds decrease with the increase of temperature.
\end{abstract}

Keywords: Ecotypes, Temperature and Scarification.

\footnotetext{
${ }^{1}$ Recebido em 13.05.2008 e aceito para publicação em 02.03.2010.

${ }^{2}$ Fundação Centro Tecnológico de Minas Gerais, CETEC, Brasil. E-mail: <renatademeloferreira@yahoo.com.br>e <valeria.freitas@cetec.br>.

${ }^{3}$ Universidade Federal de Minas Gerais, UFMG, Brasil. E-mail: <lemos@icb.ufmg.br>.
} 


\section{INTRODUÇÃO}

O gênero Plathymenia Benth. (Fabaceae, Mimosoideae) é representado por árvores tropicais nativas da América do Sul, sendo encontrado no Brasil, Bolívia, Norte do Paraguai e Suriname (LEWIS e WARWICK, 2003). Foi primeiramente descrito por George Bentham em 1842, que nele incluiu duas únicas espécies, $P$. reticulata e $P$. foliolosa (HERINGER e FERREIRA, 1972). Warming, em 1908, foi o primeiro a observar o caráter vicariante das mesmas, afirmando ser $P$. reticulata árvore campestre, dos cerradões e cerrados e $P$. foliolosa árvore de ambientes florestais (MARINIS, 1966). Ainda que Lewis e Warwick (2003) tenham considerado o gênero como monoespecífico, assumindo somente a espécie P. reticulata, diferenças ecológicas marcantes ocorrem entre os biótipos do Cerrado e da Floresta Atlântica. Dessa forma, neste trabalho continuamos a considerar duas espécies, $P$. foliolosa e $P$. reticulata.

Plathymenia reticulata Benth. é conhecida como vinhático-do-campo ou vinhático-do-cerrado, ocorre em formações abertas do Cerrado brasileiro, desde o Amapá até São Paulo, sendo encontrada em todos os estados da região Centro Oeste (ALMEIDA et al., 1998). Os indivíduos possuem de 6 a $12 \mathrm{~m}$ de altura e de 30 a $50 \mathrm{~cm}$ de diâmetro de tronco (LORENZI, 2002). Estudos sobre a germinação das sementes dessa espécie, caracterizando a ocorrência de dormência tegumentar, foram realizados por Lacerda et al. (2004) apenas na temperatura constante de $28^{\circ} \mathrm{C}$. P. foliolosa Benth., popularmente conhecida como vinhático-da-mata, estende-se no território brasileiro pela Floresta Atlântica, de São Paulo a Pernambuco, porém com maior frequência no Espírito Santo, Minas Gerais e Rio de Janeiro (RIZZINI, 1978). Os indivíduos chegam a alcançar $30 \mathrm{~m}$ de altura e mais de $70 \mathrm{~cm}$ de diâmetro de tronco (LORENZI, 2002). Não são conhecidos estudos sobre a germinação dessa espécie em condições de laboratório.

Considerando que ainda não há completo esclarecimento sobre o status taxonômico dos biotipos de Plathymenia da Floresta Atlântica e do Cerrado, dados biométricos de frutos e sementes coletados de populações desses dois biomas podem contribuir para o esclarecimento da taxonomia desse gênero. Além disso, é importante verificar a existência de respostas diferenciais no comportamento germinativo das sementes provenientes desses dois ambientes distintos, bem como comparar as respostas aos tratamentos prégerminativos para a quebra de dormência e o efeito de diferentes temperaturas e fotoperíodo na germinação. Assim, este estudo teve como objetivos comparar características biométricas de frutos e sementes e o efeito de diferentes escarificações, temperaturas e luz na germinação de Plathymenia reticulata Benth. e Plathymenia foliolosa Benth.

\section{MATERIAL E MÉTODOS}

Frutos de 10 plantas de Plathymenia reticulata, com cerca de $8 \mathrm{~m}$ de altura, foram coletados ao acaso na copa das árvores de uma mesma população, em agosto de 2003, em fragmento de Cerrado, próximo ao Município de Papagaio, MG (19¹8'S, 44³6'W). Frutos de 10 plantas de uma mesma população de $P$. foliolosa, de aproximadamente $20 \mathrm{~m}$ de altura, foram coletados no chão, sob a copa, em agosto de 2003, em um fragmento de Floresta Atlântica próximo ao Município de Bom Jesus do Amparo, MG (1942'S, $\left.43^{\circ} 30^{\prime} \mathrm{W}\right)$. Os frutos de ambas as espécies foram coletados de indivíduos que apresentavam no mínimo 10 m de distância entre si.

Na sequência foi realizada a biometria de 100 frutos e 100 sementes, desprovidas dos artículos endocárpicos (sementes nuas), para cada espécie, com uso de paquímetro, sendo registrados: comprimento, largura e espessura. Para a medida do comprimento dos frutos foram incluídos seus pedúnculos; a largura e espessura, tanto das sementes quanto dos frutos, foram obtidas da linha mediana. A massa da matéria fresca das sementes e a massa da matéria fresca dos frutos foram obtidas com uso de balança de precisão. Foram registrados, visualmente, os números de sementes intactas por fruto, predadas ou malformadas.

Lotes de quatro repetições de 10 sementes por espécie foram acondicionados em papel-alumínio, assim permanecendo sob aquecimento em estufa com temperatura controlada de $80^{\circ} \mathrm{C}$ até massa constante. O cálculo do teor de água das sementes foi feito com base em matéria seca: (massa da matéria fresca - massa da matéria seca) x 100/massa da matéria seca.

A viabilidade das sementes foi determinada utilizando-se três repetições de 40 sementes de Plathymenia reticulata e três repetições de 16 sementes de $P$. foliolosa, com delineamento experimental inteiramente casualizado. Para tal, as 
sementes foram escarificadas mecanicamente com lixa d'água $n^{\circ} 120$ na porção oposta ao eixo embrionário e colocadas para embeber em solução de cloreto de trifenil tetrazólio, onde permaneceram por $48 \mathrm{~h}$ no escuro, em germinador a $25^{\circ} \mathrm{C}$. Foram consideradas mortas as sementes em que os embriões não coloriram ou aquelas que apresentaram mais de $50 \%$ de sua estrutura sem coloração, de acordo com as Regras para Análise de Sementes (BRASIL, 1992). Para esse teste, foram excluídas as sementes malformadas e as predadas.

Quanto à germinação, foram testados dois tratamentos para a quebra da dormência das sementes, previamente desprovidas dos artículos endocárpicos: 1) escarificação mecânica com lixa d'água n 120 e 2) escarificação química com ácido clorídrico 35\%, durante 15 min, seguida de lavagem em água corrente. A desinfestação das sementes, com exceção das escarificadas quimicamente, foi realizada com solução de hipoclorito de sódio 5\%, durante 15 min, seguida de lavagem em água corrente. Foram avaliadas diferentes temperaturas constantes: 20,25 , 30 e $35^{\circ} \mathrm{C}$ e temperatura alternada de $35 / 15^{\circ} \mathrm{C}$, em fotoperíodo de $12 \mathrm{~h}$, sendo a temperatura mais elevada referente ao período de luz. Também foi utilizado o escuro contínuo em todas as temperaturas, exceto 25 e $35^{\circ} \mathrm{C}$. Em cada condição foram utilizadas quatro repetições de 25 sementes, para ambas as espécies e tratamentos, incluindo o grupo-controle. O delineamento experimental utilizado foi inteiramente casualizado. As sementes foram colocadas em caixas gerbox, sobre papel-filtro, e levadas aos germinadores de sementes, sob temperaturas constantes, e ao germinador sob temperaturas alternadas, durante 21 dias. O início das observações deu-se no terceiro dia após a implantação do experimento, sendo considerado germinação a emissão da radícula.

Os dados de biometria, massa da matéria fresca, porcentagem de sementes viáveis e de umidade das sementes foram logaritimizados (log10) para atingirem normalidade e homocedasticidade e comparados pelo teste $t$, de Student. Aqueles que não alcançaram normalidade e homocedasticidade foram comparados pelo teste U, de Mann-Whitney. Os resíduos das médias das porcentagens de germinação apresentaram distribuição normal e homocedástica e foram analisados por ANOVA, seguida de teste de comparação de médias por Tukey (FERREIRA e BORGHETTI, 2004). Todos os testes foram realizados de acordo com Zar (1996), adotando-se o nível de significância de 5\% e utilizando o programa JMP5 (SAS INSTITUTE, 2002).

\section{RESULTADOS E DISCUSSÃO}

Os frutos de Plathymenia foliolosa apresentaram diferenças significativas em relação aos frutos de $P$. reticulata para todas as variáveis estudadas ( $\leq \leq 0,001$ ), exceto para o comprimento (Tabela 1, Figura 1 ). Estudos anteriores mostraram que os frutos de $P$. foliolosa são mais compridos do que os de P. reticulata (HERINGER e FERREIRA, 1972; ALMEIDA et al., 1998). Apesar de neste trabalho não ter sido encontrada diferença significativa entre o comprimento dos frutos das duas espécies, é importante salientar que os frutos de $P$. foliolosa tiveram comprimento com maior coeficiente de variação, assim como as demais dimensões (Tabela 1). Esse fato pode ser responsável pela similaridade encontrada no comprimento dos frutos dessas duas espécies. Goulart et al. (2006) também não constataram diferenças estatísticas entre comprimento dos frutos de $P$. reticulata (considerando-se o gênero monoespecífico) oriundos de diferentes populações da Floresta Atlântica e do Cerrado.

As sementes de Plathymenia foliolosa apresentaram diferenças significativas ( $\mathrm{p} \leq 0,001)$ em relação às de $P$. reticulata, nos valores de comprimento e espessura (Tabela 1, Figura 1). Contudo, não houve diferenças na massa da matéria fresca e seca entre as duas espécies (Tabela 1). Goulart et al. (2006) não encontraram diferenças significativas entre a massa da matéria fresca e tamanho de sementes de $P$. reticulata provenientes de diferentes populações dos biomas Cerrado e Floresta Atlântica, mas verificaram que as sementes dos indivíduos da Floresta Atlântica são mais compridas, conforme os resultados deste estudo. Da mesma forma que nos frutos, as dimensões das sementes de $P$. foliolosa apresentaram maiores coeficientes de variação do que as de $P$. reticulata, exceto para massa da matéria fresca, que teve coeficiente de variação pouco menor (Tabela 1).

Os frutos de Plathymenia reticulata apresentaram 7 a 15 sementes por fruto, com média e desvio-padrão de $0,8 \pm 1,5$ semente malformada por fruto. Nos frutos

Revista Árvore, Viçosa-MG, v.34, n.5, p.797-805, 2010 
Tabela 1 - Valores mínimos, médias seguidas dos desvios-padrão (dp), valores máximos e coeficiente de variação (CV) de comprimento, largura, espessura e massas fresca e seca dos frutos (medidas em cm e g) e sementes (medidas em mm e g) de Plathymenia reticulata Benth. e Plathymenia foliolosa Benth., coletados nos Municípios de Papagaio, MG, e Bom Jesus do Amparo, MG, respectivamente.

Table 1 - Minimum, means with standard deviation (dp), maximum values and variation coefficient (CV) for length, widdth, thickness, fresh and dry mass of fruits ( $\mathrm{cm}$ and $\mathrm{g}$ ) and seeds ( $\mathrm{mm}$ and $\mathrm{g}$ ) of Plathymenia reticulata Benth. and Plathymenia foliolosa Benth., collected in the municipalities of Papagaio, MG and Bom Jesus do Amparo, MG, respectively.

\begin{tabular}{|c|c|c|c|c|c|c|}
\hline \multicolumn{7}{|c|}{ Frutos } \\
\hline Espécies & Valores & Comprimento & Largura & Espessura & Massa Fresca & Massa Seca \\
\hline P. reticulada & $\begin{array}{c}\text { Mín. } \\
\text { Média + d.p. } \\
\text { Máx. } \\
\text { CV }\end{array}$ & $\begin{array}{c}13,05 \\
17,62 \pm 1,66 \mathrm{~A} \\
21,11 \\
9,41\end{array}$ & $\begin{array}{c}1,95 \\
2,51 \pm 0,18 \mathrm{~B} \\
2,94 \\
7,26\end{array}$ & $\begin{array}{c}0,20 \\
0,29 \pm 0,05 \mathrm{~B} \\
0,42 \\
16,67\end{array}$ & $\begin{array}{c}1,80 \\
3,40 \pm 0,67 \mathrm{~B} \\
5,15 \\
19,75\end{array}$ & $\begin{array}{l}- \\
- \\
-\end{array}$ \\
\hline P. foliodosa & $\begin{array}{c}\text { Mín. } \\
\text { Média + d.p. } \\
\text { Máx. } \\
\text { CV }\end{array}$ & $\begin{array}{c}9,20 \\
16,85 \pm 3,85 \mathrm{~A} \\
27,50 \\
22,83\end{array}$ & $\begin{array}{c}2,24 \\
2,89 \pm 0,41 \mathrm{~A} \\
3,70 \\
14,07\end{array}$ & $\begin{array}{c}0,20 \\
0,32 \pm 0,10 \mathrm{~A} \\
0,70 \\
31,37\end{array}$ & $\begin{array}{c}1,48 \\
3,97 \pm 1,49 \mathrm{~A} \\
7,63 \\
37,47\end{array}$ & $\begin{array}{l}- \\
- \\
-\end{array}$ \\
\hline & & & Sementes & & & \\
\hline Espécies & Valores & Comprimento & Largura & Espessura & Massa Fresca & Massa Seca \\
\hline P. reticulada & $\begin{array}{c}\text { Mín. } \\
\text { Média + d.p. } \\
\text { Máx. } \\
\text { CV }\end{array}$ & $\begin{array}{c}6,30 \\
7,79 \pm 0,74 \mathrm{~b} \\
9,40 \\
9,54\end{array}$ & $\begin{array}{c}4,60 \\
6,16 \pm 0,68 a \\
7,70 \\
10,97\end{array}$ & $\begin{array}{c}1,20 \\
1,54 \pm 0,17 \mathrm{a} \\
2,00 \\
11,01\end{array}$ & $\begin{array}{c}23,40 \\
0,048 \pm 0,001 \mathrm{a} \\
76,60 \\
23,93\end{array}$ & $\begin{array}{c}0,04 \\
0,041 \pm 0,002 \mathrm{a} \\
0,04 \\
6,17\end{array}$ \\
\hline P. foliodosa & $\begin{array}{c}\text { Mín. } \\
\text { Média + d.p. } \\
\text { Máx. } \\
\text { CV }\end{array}$ & $\begin{array}{c}5,90 \\
8,46+1,21 \mathrm{a} \\
10,40 \\
14,35\end{array}$ & $\begin{array}{c}3,80 \\
6,18 \pm 1,07 a \\
8,50 \\
17,34\end{array}$ & $\begin{array}{c}1,00 \\
1,44+0,17 \mathrm{~b} \\
1,80 \\
11,72\end{array}$ & $\begin{array}{c}21,20 \\
0,046+0,001 \mathrm{a} \\
64,4 \\
21,89\end{array}$ & $\begin{array}{c}0,04 \\
0,048 \pm 0,003 a \\
0,05 \\
7,18\end{array}$ \\
\hline
\end{tabular}

Médias seguidas de letras distintas nas colunas (maiúsculas para frutos e minúsculas para sementes) diferem entre si, pelo teste t, de Student, ou teste U, de Mann-Whitney.

Means followed by distinct letters in columns (capital letters for fruits and small caps for seeds) differed accordingly to the Student or Mann-Whitney U tests.

de $P$. foliolosa, foram encontradas de 3 a 19 sementes por fruto, sendo 2,6 \pm 2,6 sementes malformadas por fruto (Tabela 2). Foi observado que as sementes localizadas próximas às extremidades do fruto ou nas suas reentrâncias eram menores e mais sujeitas ao aborto, tanto em $P$. reticulata quanto em $P$. foliolosa. A porcentagem de sementes de P. reticulata predadas por coleópteros (Família Bruchinae) foi inferior $(\mathrm{p}=0,03)$ em relação à das sementes de P. foliolosa (Tabela 2). Os dados de coeficiente de variação indicaram que, em ambas as espécies, as quantidades de sementes malformadas e predadas por fruto foram muito variáveis.

Houve diferença significativa $(p=0,03)$ no teor (\%) de água das sementes das duas espécies estudadas (Tabela 2). Entretanto, essa diferença não afetou a viabilidade, pois o teste do tetrazólio indicou alta porcentagem de sementes viáveis em Plathymenia reticulata, assim como em $P$. foliolosa (Tabela 2).
A germinação iniciou-se no terceiro dia, e as sementes que não foram escarificadas germinaram ao longo de 21 dias, enquanto as escarificadas concentraram esse evento nos três primeiros dias do teste.

As porcentagens de germinação das sementes intactas aumentaram com a temperatura $(\mathrm{p} \leq 0,001)$ (Tabela 3, Figura 2). Em $P$. reticulata, 93\% das sementes intactas germinaram a $30^{\circ} \mathrm{C}$ e $55 \%$ a $25^{\circ} \mathrm{C}$, em ambos os casos sob fotoperíodo de $12 \mathrm{~h}$. As sementes não escarificadas de $P$. foliolosa apresentaram maior germinabilidade a partir de $30^{\circ} \mathrm{C}(12 / 12 \mathrm{~h})$, com valores superiores a 62\% de germinação (Tabela 3, Figura 2).

Para Plathymenia reticulata, as porcentagens de germinação das sementes intactas foram tão altas quanto das sementes escarificadas química e mecanicamente, em todas as temperaturas, exceto $25^{\circ} \mathrm{C}$ (Tabela 3, Figura 2). Lacerda et al. (2004), estudando diferentes populações de $\mathrm{P}$. reticulata, verificaram valores de germinabilidade similares, com as sementes intactas germinando entre 40 e $60 \%$ a $25^{\circ} \mathrm{C}$ e as escarificadas mecanicamente com valores superiores a $84 \%$. 

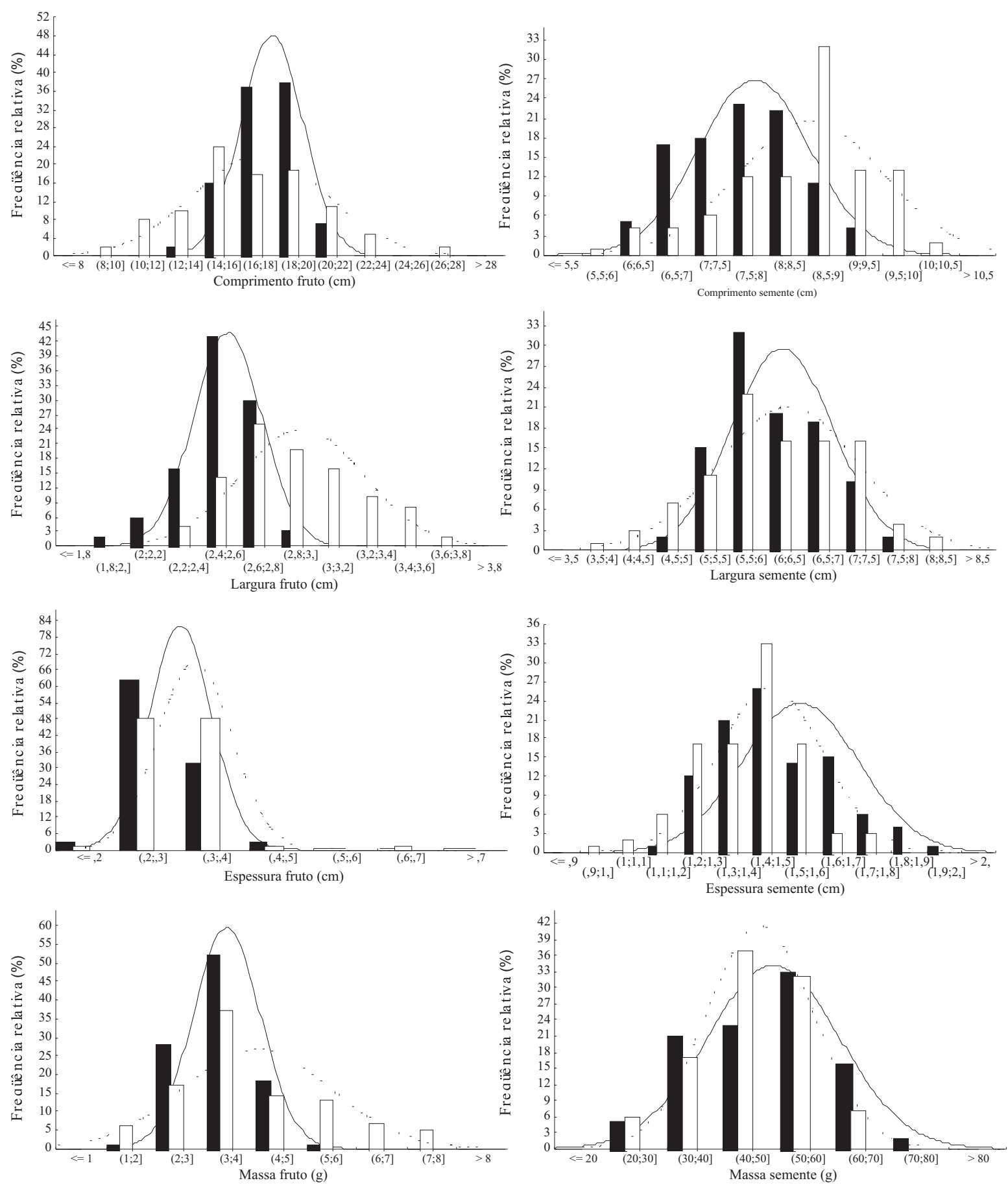

Figura 1- Frequência de massa de matéria fresca e dimensões dos frutos e sementes de Plathymenia reticulata Benth. (barras claras e curvas descontínuas) e Plathymenia foliolosa Benth. (barras escuras e curvas contínuas), coletados nos Municípios de Papagaio, MG, e Bom Jesus do Amparo, MG, respectivamente.

Figure 1 - Fresh mass frequency and fruits and seeds dimensions of Plathymenia reticulata Benth. (clear bars and dotted curves) and Plathymenia foliolosa Benth. (dark bars and continuous curves), colleted in the municipalities of Papagaio, MG and Bom Jesus do Amparo, MG, respectively. 
Tabela 2 - Média do número de sementes por fruto e aborto, predação, viabilidade e umidade (\%) das sementes de Plathymenia reticulata Benth. e Plathymenia foliolosa Benth. coletadas nos Municípios de Papagaio, MG, e Bom Jesus do Amparo, MG, respectivamente.

Table 2 - Means of the number of seeds per fruit and abort, predation, viability and humidity (\%) of seeds of Plathymenia reticulata Benth. and Plathymenia foliolosa Benth., colleted in the municipalities of Papagaio, MG and Bom Jesus do Amparo, MG, respectively.

\begin{tabular}{lcccrr}
\hline \multicolumn{5}{c}{ Sementes } \\
\hline Espécies & No/fruto & \% Abortadas & \% Predadas & \% Viáveis & \% Umidade \\
P. reticulata & $11,8+1,7 \mathrm{~A}$ & $7,2+14,1 \mathrm{~B}$ & $9,2+11,8 \mathrm{~B}$ & $95,0+0,8 \mathrm{~A}$ & $11,4+0,3 \mathrm{~A}$ \\
$P$. foliolosa & $11,4+3,5 \mathrm{~A}$ & $23,3+23,5 \mathrm{~A}$ & $15,7+25,5 \mathrm{~A}$ & $97,9+0,6 \mathrm{~A}$ & $10,1+0,8 \mathrm{~B}$ \\
\hline
\end{tabular}

Médias \pm desvio-padrão seguidas de letras distintas diferem entre si, pelo teste $t$ de Student (5\%).

Means \pm standard deviation followed by distinct letters differed accordingly to the Student $t$ test (5\%).

$\mathbf{A}$

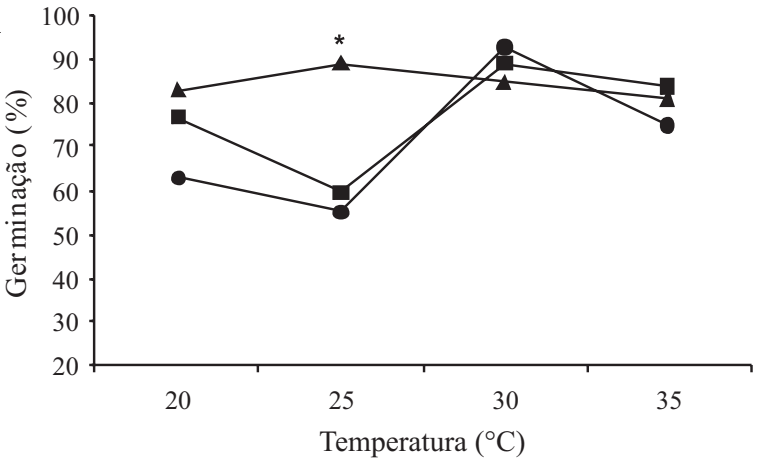

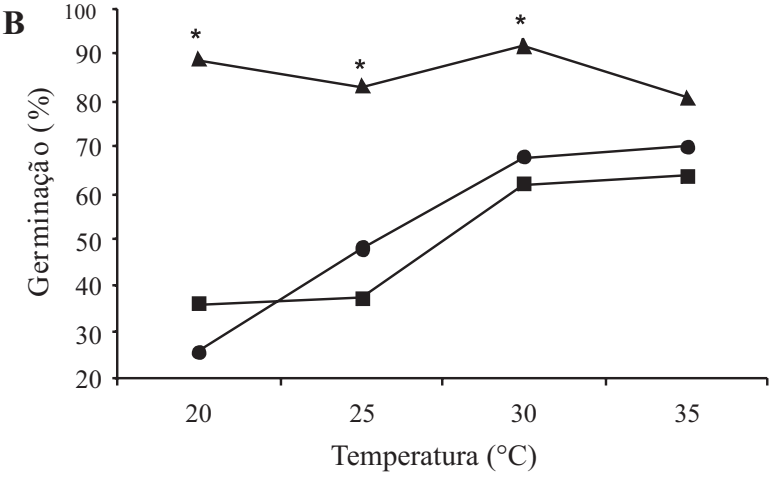

Figura 2 - Germinação (\%) das sementes de Plathymenia reticulata Benth. (A) e Plathymenia foliolosa Benth. (B) coletadas nos municípios de Papagaio, MG e Bom Jesus do Amparo, MG, respectivamente, em diferentes temperaturas testadas após terem sido submetidas aos tratamentos pré-germinativos • sementes íntegras (grupo controle); - sementes escarificadas com ácido; $\boldsymbol{\Lambda}$ sementes escarificadas com lixa. O símbolo $(*)$ indica diferença significativa entre a porcentagem de germinação indicada e as demais porcentagens, dentro da mesma temperatura.

Figure 2 - Seeds germination (\%) of Plathymenia reticulata Benth. (A) and Plathymenia foliolosa Benth. (B) collected in the municipalities de Papagaio, MG and Bom Jesus do Amparo, MG, respectively, under different temperatures and light conditions. Some groups were submitted to pre-germinative treatments. $\bullet$ seeds (control group); - seeds treated with acid; $\boldsymbol{\Lambda}$ seeds treated with sandpaper. The symbol (*) indicate significant differences between the indicated germinations percentage and the others percentages, within the same temperature.

As porcentagens de germinação das sementes intactas de Plathymenia foliolosa foram diferentes estatisticamente das escarificadas mecanicamente e não das escarificadas com ácido, em todas as temperaturas (Tabela 3, Figura 2). Esses dados indicam que o lote de sementes de $P$. foliolosa apresentou tegumento mais duro, comparativamente às sementes de P. reticulata. Outros autores, utilizando concentrações de ácido mais elevadas, obtiveram altas porcentagens de germinação de sementes de leguminosas, a exemplo de Lopes et al. (2006), que verificaram 96\% de germinação de sementes de Ormosia nitida Vog. escarificadas com ácido sulfúrico durante $15 \mathrm{~min}$, à temperatura e luminosidade ambientes;
Antônio et al. (1985), que verificaram 100\% de germinação de sementes de Galactia striata (Jacq.) Urban submetidas a ácido sulfúrico concentrado durante $15 \mathrm{~min}$, a $30^{\circ} \mathrm{C}$, no escuro; e Scheffer-Basso e Vendrusculo (1997), que obtiveram 84\% de sementes germinadas de Adesmia araujoi Burkart submetidas a ácido sulfúrico 95\% durante $1 \mathrm{~min}$, a $25^{\circ} \mathrm{C}$, no claro.

Com relação à alternância de temperaturas, não foram observadas diferenças na porcentagem final de germinação das sementes intactas e das escarificadas e submetidas às temperaturas constantes, em comparação com as temperaturas alternadas (Tabela3). 


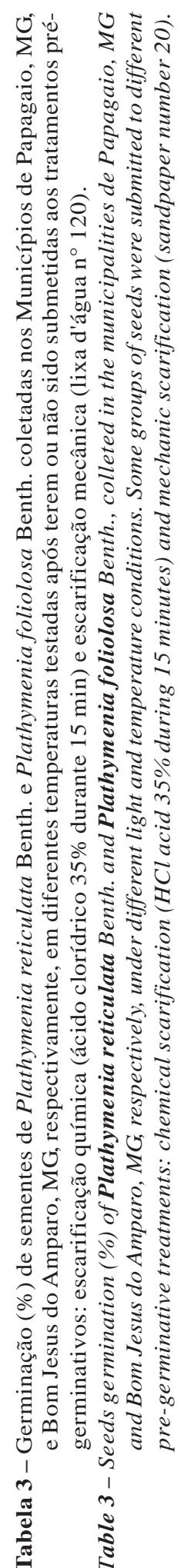

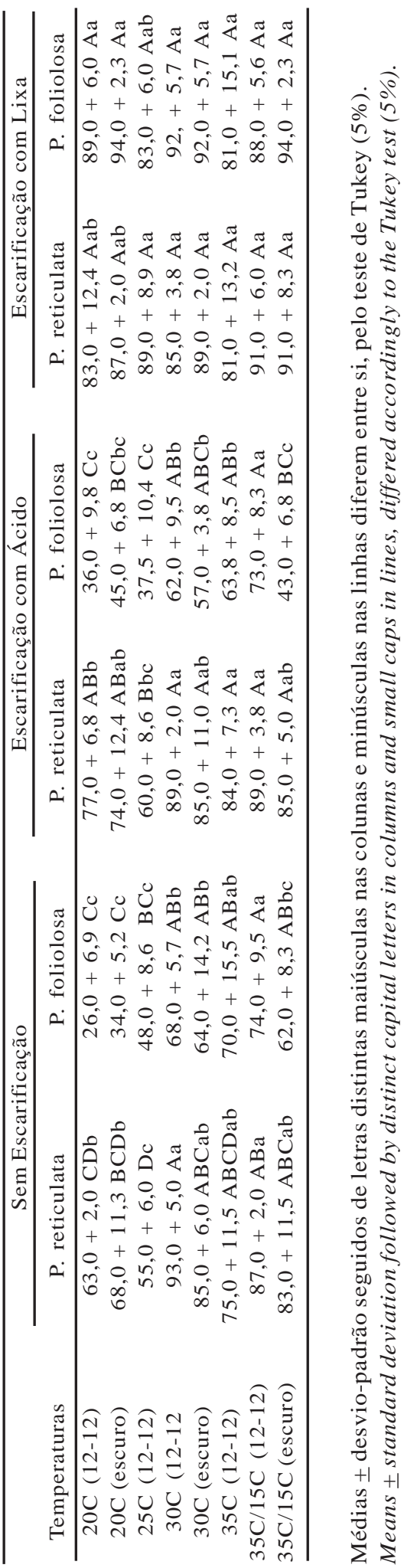

As sementes de ambas as espécies também não se apresentaram fotossensíveis. Esse resultado indica que as sementes podem germinar em clareiras ou subbosques, o mesmo observado por Aguiar et al. (2005) em sementes da leguminosa Caesalpinia echinata Lam. (pau-brasil).

Lesões provocadas pela escarificação mecânica podem ter favorecido a atividade de fungos, de maneira que, se as sementes não germinassem aproximadamente nos três primeiros dias de experimento, elas se apresentariam deterioradas e totalmente encobertas por micélios de fungos. A escarificação química mostrouse adequada como agente de desinfestação, já que a atividade fúngica sobre as sementes submetidas a esse tratamento foi menor. A escarificação manual, por sua vez, apesar de demandar mais tempo, permite que se obtenha germinação mais homogênea e sincrônica, o que é desejável para a produção de mudas, além de que não incorre em danos ao meio ambiente, como os causados pelos resíduos da utilização de ácidos em geral.

Cunha et al. (1990) encontraram que sementes de Couroupita guianensis Aubl. (Lecythidaceae) provenientes de frutos maduros ainda fechados e coletados das árvores apresentaram valores de germinação significativamente maiores que os de sementes originárias de frutos rachados e coletados imediatamente após sua queda. Neste estudo, as sementes dos dois lotes foram obtidas de forma distinta, as de $P$. reticulata de frutos ainda na copa e as de $P$. foliolosa a partir de frutos recém-dispersos. Portanto, não se pode descartar a possibilidade de que as diferenças observadas na germinaçao tenham relação com esse fato.

Segundo Vázquez-Yanes e Orozco-Segovia (1993), as sementes em geral possuem características morfológicas e fisiológicas que refletem suas respostas às pressões seletivas do ambiente onde se desenvolvem. Entretanto, Goulart et al. (2005) refutaram parcialmente essa afirmação ao constatarem que Plathymenia apresenta comportamento fenológico semelhante no Cerrado e na Floresta Atlântica, sendo apenas $10 \%$ da diversidade fenológica atribuída aos diferentes biomas.

Revista Árvore, Viçosa-MG, v.34, n.5, p.797-805, 2010 


\section{CONCLUSÕES}

Os dados biométricos obtidos no presente estudo confirmam as diferenças relatadas por Heringer (1956 apud LEWIS E WARWICK, 2003) e Bentham (1842 apud HERINGER E FERREIRA, 1972) que reconhecem Plathymenia reticulata e $P$. foliolosa como espécies distintas. O comportamento germinativo diferencial das sementes também aponta para esse fato. Esses resultados indicam que, se não espécies distintas, é possível que sejam ecótipos diferenciados de Plathymenia do Cerrado e da Floresta Atlântica.

\section{AGRADECIMENTOS}

Aos professores Marcel Geovanni C. França(UFMG) e Juliana L. P. Rezende (PUC-Betim), pela leitura do manuscrito, a David Bird (UQAM-Montreal) e Fernando A. O. Silveira (UFMG), pelo auxílio nas análises estatísticas, à Maíra F. Goulart (UFMG) pelo auxílio nas coletas de sementes, à Janaína Pizzatti (UFOP-MG), pela identificação dos coleópteros, aos técnicos e bolsistas do Lab. Ecofisiologia Vegetal do CETEC-MG e à FAPEMIG, pelo financiamento do projeto e concessão de bolsa de iniciação científica à primeira autora.

\section{REFERÊNCIAS}

AGUIAR, F. F. A. et al. Germinação de sementes e formação de mudas de Caesalpinia echinata Lam. (pau-brasil): efeito de sombreamento. Revista Árvore, v.29, n.6, p.871-875, 2005.

ALMEIDA, S. P. et al. Cerrado: Espécies Vegetais Úteis. Planaltina, EMBRAPA-CPAC, 1998. 456p.

ANTÔNIO, F. G.; PENTEADO, M. I. O.; SEIFFERT, N. F. Recomendações para Quebra de Dormência em Sementes de Galactia spp. Comunicado Técnico no. 29, Campo Grande, EMBRAPA Gado de Corte, p.8, 1985.

BRASIL. Regras para Análise de Sementes. Ministério da Agricultura e da Reforma Agrária, Secretaria Nacional de Defesa Agropecuária. Departamento Nacional de Defesa Vegetal. Brasília, 1992. 365p.

CUNHA, R. et al. Secagem, desinfestação e germinação de sementes de Couroupita guyanensis Aub. Revista Brasileira de Sementes, v.12, n.2, p.74-79, 1990.
FERREIRA, A. G.; BORGHETTI, F. Germinação: do básico ao aplicado. Porto Alegre, Artmed, 2004. 324p.

GOULART, M. F.; LEMOS-FILHO, J. P.; LOVATO, M. B. Phenological variation within and among populations of Plathymenia reticulata in brazilian Cerrado, the Atlantic Forest and transitional sites. Annals of Botany, v.96, n.3, p.445-455, 2005.

GOULART, M. F.; LEMOS-FILHO, J. P.; LOVATO, M. B. Variability in fruit and seed morphology among and within populations of Plathymenia (Leguminosae-Mimosoideae) in areas of the Cerrado, the Atlantic Forest and transitional sites. Plant Biology, v.8, n.1, p.112-119, 2006.

HERINGER, E. P.; FERREIRA, M. B. Árvores úteis no Cerrado: (I) Vinhático - o gênero Plathymenia Benth. P. foliolosa Benth. e P. reticulata Benth., vinhático-da-mata e vinhático-do-campo (par vicariante). Cerrado, v.5, p.28-34, 1972.

LACERDA, D. R. et al. Seed-dormancy variation in natural populations of two tropical leguminous tree species: Senna multijuga (Caesalpinoideae) and Plathymenia reticulata (Mimosoideae). Seed-Science-Research, v.14, n.2, p.127-135, 2004.

LEWIS, G. P.; WARWICK, M. C. Revision of Plathymenia (Leguminosae-Mimosoideae).

Endinburgh Journal of Botany, v.60, n.2, p.111-119, 2003.

LOPES, J. C.; DIAS, P. C.; MACEDO, C. M. P. Tratamentos para acelerar a germinação e reduzir a deterioração das sementes de Ormosia nitida Vog. Revista Árvore, v.30, n.2, p.171-177, 2006.

LORENZI, H. Árvores Brasileiras. Manual de identificação e cultivo de plantas arbóreas do Brasil. Instituto Plantarum, Nova Odessa, vol.1, 2002. 368p.

MARINIS, G. Contribuição ao conhecimento das espécies vicariantes do gênero Plathymenia. Anais da Academia Brasileira de Ciências, São José do Rio Preto, v.38 (suplemento), p.71-83, 1966.

RIZZINI, C. T. Árvores e Madeiras Úteis do Brasil - Manual de Dendrologia Brasileira. São Paulo: Edgard Blücher Ltda, 1978. 296p. 
SAS INSTITUTE. JMP 5.0. A Business Unit of SAS. 2002.

SCHEFFER-BASSO, S. M.; VENDRUSCULO, M. C. Germinação de Sementes das Leguminosas Forrageiras ativas Adesmia araujoi Burk. e Desmodium incanum D.C. Revista Brasileira de Agrociência, v.3, n.2, p.65-68, 1997.
VÁZQUEZ-YANES, C.; OROZCO SEGOVIA, A. Patterns of seed longevity and germination in the tropical rainforest. Annual Review of Ecology and Systematics, v.24, p.69-87, 1993.

ZAR, J. H. Biostatistical Analysis. London, Prentice-Hall International, Inc., 1996. 662p. 